\title{
A UK multicentre retrospective cohort study comparing hysterectomy and uterine artery embolisation for the treatment of symptomatic uterine fibroids (HOPEFUL study): main results on medium-term safety and efficacy
}

\author{
S Dutton, a A Hirst, ${ }^{b}$ K McPherson, ${ }^{c}$ T Nicholson, $^{d}$ M Mareshe \\ ${ }^{a}$ Centre for Statistics in Medicine, Wolfson College, University of Oxford and ${ }^{\mathrm{b}}$ CTSU, University of Oxford, Oxford, UK \\ ' Nuffield Department of Obstetrics and Gynaecology, John Radcliffe Hospital, Oxford, UK d Department of Radiology, Leeds General \\ Infirmary, Leeds, UK ${ }^{\mathrm{e}}$ Department of Obstetrics and Gynaecology, St Mary's Hospital for Women and Children, Central Manchester \\ and Manchester Children's University Hospitals NHS Trust, Manchester, UK \\ Correspondence: Mrs S Dutton, Centre for Statistics in Medicine, Wolfson College, University of Oxford, Linton Road, Oxford OX2 60D, UK \\ Email susan.dutton@cancer.org.uk
}

Accepted 21 August 2007.

Objectives Comparison of medium-term safety and efficacy of hysterectomy and uterine artery embolisation (UAE) for symptomatic uterine fibroids.

Design Multicentre retrospective cohort.

Setting 18 UK NHS hospital trusts.

Participants Four hundred and fifty nine women who had hysterectomy within a national audit during 12 months from October 1994 (VALUE study) (average follow up of 8.6 years) and 649 women receiving UAE from 1996 to 2002 (average follow up of 4.6 years).

Methods Clinical data from existing hospital records and patient completed postal questionnaires.

Main outcome measures Complication rates, side effects of embolisation, satisfaction with treatment, relief from symptoms and requirement for further fibroid treatment.

Results Fewer complications were experienced by women receiving UAE ( 19 versus $26 \%$ hysterectomy, $P=0.001$ ), the adjusted odds ratio for UAE versus hysterectomy was 0.48 (95\% CI 0.26-0.89).
One-third of women undergoing UAE experienced anticipated general side effects associated with the procedure. More women in the hysterectomy cohort reported relief from fibroid symptoms (95 versus $85 \%, P<0.0001$ ) and feeling better (96 versus $84 \%$, $P<0.0001$ ), but only $85 \%$ would recommend the treatment to a friend compared with $91 \%$ in the UAE arm $(P=0.007)$. There was a $23 \%(95 \%$ CI $19-27 \%)$ chance of requiring further treatment for fibroids after UAE. Twenty-seven women who had had UAE reported 37 pregnancies after treatment resulting in 19 live births.

Conclusions UAE results in fewer complications than hysterectomy. Side effects after embolisation should be anticipated, and almost one-quarter of women having UAE were likely to require further treatment for fibroid symptoms. Both treatments appear to be safe and effective over the medium term, and the choice of treatment may be a matter of personal preference for each individual woman.

Keywords Complications, embolisation, hysterectomy, uterine fibroids.

Please cite this paper as: Dutton S, Hirst A, McPherson K, Nicholson T, Maresh M. A UK multicentre retrospective cohort study comparing hysterectomy and uterine artery embolisation for the treatment of symptomatic uterine fibroids (HOPEFUL study): main results on medium-term safety and efficacy. BJOG 2007;114:1340-1351.

\section{Introduction}

Uterine fibroids (leiomyomata) are benign tumours of smooth muscle cells and fibrous connective tissue that develop within the wall of the uterus, occurring with clinical significance in $20-40 \%$ of women of childbearing age. ${ }^{1}$ The aetiology and pathogenesis of fibroids is not fully understood, but their occurrence during the female reproductive lifespan indicates an association with the hormones estrogen and progesterone. Prevalence of clinically significant fibroids peaks in 
the perimenopausal years declining after the menopause. ${ }^{2}$ Most fibroids cause no symptoms and are an incidental finding during a clinical or ultrasound examination. Such fibroids require no treatment other than monitoring. However, uterine fibroids are responsible for significant morbidity in the female population. The most common symptoms are associated with menstruation and include heavy menstrual bleeding (menorrhagia), which may lead to iron deficiency anaemia and dysmenorrhoea. Fibroids also cause a variety of other symptoms due to their bulk or pressure upon adjacent organs, particularly the bladder, causing varying urinary symptoms.

No pharmacological intervention is known to have a longterm effect on symptoms of fibroids, ${ }^{3}$ although it is possible to use gonadotrophin-releasing hormone agonists with addback therapy in the short to medium term in specific instances. The standard treatment for symptomatic uterine fibroids has been hysterectomy (surgical removal of the uterus, including the fibroid[s]). Myomectomy (surgical removal of the fibroid[s] only) has also been an alternative treatment for symptomatic fibroids. During the mid-1990s, a minimally invasive uterus-conserving treatment was described known as uterine artery embolisation (UAE). ${ }^{4}$ Evidence from a few small randomised controlled trials suggests that UAE is a safe and effective treatment up to 12 months, with a shorter hospital stay and quicker resumption to daily activities than hysterectomy. ${ }^{5-11}$ The US fibroid registry was established to examine long-term effects of UAE prospectively and early results with 12 month follow up have led the authors to conclude that UAE is a low-risk procedure with little variability in adverse events based on patient demographics or practice setting. ${ }^{12-15}$

The long-term safety and efficacy of UAE remains poorly understood. ${ }^{16-19}$ The HOPEFUL (Hysterectomy Or Percutaneous Embolisation For Uterine Leiomyomata) study was commissioned by the UK NHS Health Technology Assessment (HTA) programme (www.hta.nhsweb.nhs.uk/) as a pragmatic study to investigate and compare UAE and hysterectomy retrospectively over the medium term with regard to safety, efficacy, cost-effectiveness and women's own perspectives on the treatments. A full report is in preparation for publication by the HTA in early $2008 .{ }^{20}$ Cost-effectiveness analyses $^{21}$ and qualitative analyses are described in that report. The objective of this paper was to report the study methods and main results related to the quantitative data comparing safety and efficacy of the two treatments.

\section{Methods}

The HOPEFUL study is a pragmatic multicentre retrospective cohort design comparing the experiences of two representative cohorts of women who received one of two alternative treatments for symptomatic fibroids from the mid-1990s. There was no exclusions by age, other medical conditions or any other variable, as the study population was intended to represent
UK experience at that time. The hysterectomy cohort underwent their index treatment in the 12 months beginning from October 1994 as part of a national audit of hysterectomies in England (VALUE study). ${ }^{23}$ These women were recruited from the nine VALUE centres that had performed the most hysterectomies for uterine fibroids to maximise patient numbers and for pragmatic reasons to minimise the number of centres for hospital-based data collection. The majority of the hysterectomies were total abdominal $(86.7 \%)$, with the remainder being subtotal (5.0\%), vaginal (5.2\%) or laparoscopically assisted vaginal $(2.6 \%)$. There was insufficient power to analyse these small subgroups separately. The women who underwent UAE were treated in ten centres (nine in England and one in Scotland) by interventional radiologists who had pioneered its use since 1996 and all received their index UAE prior to the end of 2002. We were able to locate 1734 eligible women, 972 treated with embolisation and 762 with hysterectomy.

All consultants responsible for the patients identified either in the hysterectomy or in the UAE cohorts acted as local Principal Investigators (PI) for the study. A formal participation agreement was signed for each centre between the local PIs, the participating NHS Trust R\&D Directors and the University of Oxford. Temporary researchers were employed within each of the collaborating hospitals to collect clinical data according to the study protocol.

Overall ethical approval for the HOPEFUL study was obtained from the Multicentre Research Ethics Committee (MREC) for Scotland, Edinburgh. (REC reference MREC/ 03/10/49). Full approval was received on 23 February 2004. Subsequent approvals for small amendments to the protocol were sought and obtained. In addition to the overall MREC approval for the project, each local centre obtained their Local Research Ethics Committee site-specific approval and local NHS Trust R\&D Management approval prior to start of the study.

Participants' consent was obtained prior to any data collection, and confidentiality was maintained by using a passwordprotected patient database on a separate PC to the main HOPEFUL database. Patient ID numbers were allocated and used in the main project database to maintain anonymity.

Clinical data only were collected for the 28 deceased women (hysterectomy, $n=21$; UAE, $n=7$ ). The further 1706 living women were invited to consent by post (hysterectomy, $n=762$; UAE, $n=972$ ) and reminded if they had not responded within 4 weeks of the first invitation. All consenting women and deceased women were included in the study. Nine hundred and thirty-four (55\%) women responded to this first call, and a further 160 (9\%; hysterectomy, $n=60$; UAE, $n=100$ ) women responded to the second invitation (772 letters sent; hysterectomy, $n=360$; UAE, $n=412$ ). Overall, 64\% (1094) of the living eligible women consented to participate (hysterectomy, $n=438$; UAE, $n=656$ ). This gave a total of 1122 women available for data collection. 
Following the receipt of each woman's signed consent by Oxford central office, the relevant local centre was requested to proceed with clinical data collection from the women's hospital notes. The clinical data forms included details confirming the diagnosis, full details of the surgery or embolisation techniques used, relevant treatment outcomes and complications and further investigations or treatments over a period of several years of follow up. Additional parts of the data forms were provided to collect data on any pregnancies after UAE or any deaths after treatment.

Issues of nonstandard data collection across centres and missing data due to local variations in hospital record keeping were identified. There were 136 women whose hospital notes could not be retrieved. Sixty-six of these were in the hysterectomy cohort, and for these women, we were able to merge basic clinical data from the VALUE database. The large number of unavailable notes in this group was due to the fact that their treatment was 10 years ago and many NHS hospital notes were destroyed after 8 years of inactivity. Also, for some centres, the hospital records had been archived on microfiche causing some difficulty in accessing information. The hospital notes of 70 women who underwent UAE were not available for examination, although most patient questionnaires had been returned. These missing clinical forms were primarily from the centre with the largest patient population, where it was not possible to complete all the clinical forms during the study period. There was no significant difference $(P=0.49)$ between numbers of complications for women with and without clinical data. Overall, 94\% of the clinical forms were completed.

At receipt of consent, a simple follow-up patient questionnaire was sent to the consenting women with a FREEPOST envelope for return to Oxford. The women were assured of their confidentiality and provided with a FREEPHONE telephone number if they had any concerns or questions while completing the questionnaire. Reminder letters and additional questionnaires were sent to women who had not returned questionnaires after 4 weeks following the first questionnaire being sent. A third questionnaire was sent to all women who had not responded to the second questionnaire reminder in November 2005. Overall, 90\% of questionnaires sent out were returned.

There are two main purposes to this study. First, to confirm previous reports on the safety of UAE compared with hysterectomy in the short term and second, to enable the mediumterm experience of a large number of women to be examined. The latter data were not generally available in clinical trials of efficacy, particularly where the known outcomes are so different and hence where preferences are likely to be strong. However, there are important methodological considerations to be addressed due to the retrospective study design. These are fully considered in the analyses and include the possible lack of comparability of the two cohorts at baseline, the dif- ficulty of identifying relevant outcome measures to enable a direct comparison between two treatments, one of which removes the uterus and thus eliminates the source of the symptoms. Furthermore, the retrospective design means no pretreatment validated measures of quality of life/symptoms is available to compare with values after treatment in both groups.

The primary outcome measure addressed comparative safety, that is negative events resulting from treatment measured as complication rates. The project team agreed a priori on the definitions regarding the categorisation of complication severity. ${ }^{20}$ The clinicians were sent a blinded list of all the complications reported on both clinical forms and questionnaires to categorise the complications into these types. A project team meeting followed this independent assessment, where agreement was reached on the final categorisation of complications into severity. Examples of some of the complications reported. Severe complication, patient A: renal failure, treated on intensive treatment unit (ITU)—categorised as 'organ failure'. Major complications, patient B: blood transfusion after stitches burst and blood loss-categorised as 'haemorrhage requiring transfusion'; patient C: bladder perforated during operation, repaired in theatre-categorised as 'structural damage caused by treatment'; patient E: urgent hysterectomy for sepsis of fibroids-categorised as 'major infection'; patient A: bronchopneumonia, return to ITU, 'major infection'. Minor complications included urinary tract infection, Escherichia coli, wound infection, haematoma, fibroid extraction (not requiring myomectomy or hysterectomy), granulated tissue to vault requiring silver nitrate cauterisation, haemorrhage not requiring transfusion, bradycardia, severe pain requiring readmission.

All severe, major and most minor complications occurred within 30 days of treatment, although some minor complications, in particular assisted fibroid extractions, occurred up to 1 year after UAE. Each woman with one or more complication was then categorised according to the most severe of their complications, for example patient A described above experienced both severe and major complications and was categorised as severe.

Some women who underwent UAE also suffered from general side effects (GSEs) of the treatment. These include postembolisation syndrome (fever, pain, nausea lasting from a few hours to a few days), vaginal discharge caused by the disintegrating fibroids, natural expulsion of the whole or parts of the fibroid (not requiring assistance) and temporary amenorrhoea.

Postembolisation syndrome is well known and well recognised by physicians who perform embolotherapy. ${ }^{23-24}$ It occurs in relation to embolisation of the liver, kidneys, spleen and other solid organs and is part of the immune response. ${ }^{25-27}$ It is easily treated with anti-inflammatory and antipyretic drugs. Indeed, it is increasingly recognised as part of the 
postprocedural effects of aortic and thoracic stent grafting secondary to the metallic stent. The discomfort associated with embolisation is easily controlled with a simple analgesia regimen. GPs would normally refer a patient back to hospital if they required more than this.

It was decided a priori by the project team on the advice of the clinicians not to include GSEs as complications. However, because other studies have considered them as complications, GSEs were also investigated and are presented here to provide the complete picture of all outcomes from both treatments. Please note that when one of these GSEs led onto a complication, the woman was categorised as having the complication. An example of this is where a chronic vaginal discharge may have led to requiring intervention to extract a disintegrating fibroid or treatment with antibiotics for a minor infection.

Secondary outcomes relate to treatment efficacy. Data were gathered retrospectively primarily by patient questionnaire on resolution of fibroid symptoms and satisfaction with treatment. In addition, any further treatments for continuing or recurrent symptoms were recorded as a further measure of efficacy in the UAE cohort only. Pregnancies after UAE and their outcomes were also recorded.

\section{Statistical analysis}

STATA software ${ }^{29}$ (Version 8) was used for all the statistical analyses.

\section{Sample size}

VALUE $^{22,30}$ reported complication rates of $3.5 \%$ for operative complications and a further $9 \%$ for postoperative complications. In addition, the complication rate was found to be 1.5 times higher for women with fibroids compared with those with dysfunctional uterine bleeding. ${ }^{30}$ The VALUE team carried out an ascertainment exercise ${ }^{22}$ to compare the data submitted by clinicians with a review of 1453 cases relating to women having hysterectomies during the same time period. These were obtained by searching the operating theatre records in randomly selected hospitals. Case note review suggested that submitted forms under-reported major operative haemorrhage and postoperative complications. As HOPEFUL uses both patient records and questionnaires for data collection, complication ascertainment is likely to be more complete and would be expected to be higher than that for VALUE. To cover this possible underreporting, it is assumed for the purpose of this sample size calculation that the rate of all complications for hysterectomy is $20 \%$. To detect a halving of the odds ratio with a power of $90 \%$ at the $5 \%$ significance level, 370 women were required to be recruited in each cohort from a total of 740 women.

The a priori confounders considered included age, educational level, ethnicity, parity, menopausal status, smoking, medical co-morbidity, prior pelvic surgery and prophylactic antibiotics (Table 1). Chi-square test statistics were used to compare these baseline characteristics between the two cohorts.

Missing values were expected to occur in some of the confounding variables due to collecting data retrospectively from different sources and different centres. Inspite of all attempts to collect complete data, there were missing data items in one or more of the a priori defined confounding variables for $53.1 \%$ of the women, although there were less than $10 \%$ missing items in total. To use all the available data, missing values were estimated using validated multiple imputation methods, ${ }^{20,32}$ on the assumption that they were missing at random. These methods provide the most unbiased estimate of the main comparison available because excluding women with any missing data not only affects the precision of the estimates but also likely to bias the results. (For further details see the HTA monograph). ${ }^{20}$

Women from different centres may be more dissimilar than those in the same centre due to known geographic variation in factors that may affect their risk of an adverse outcome. The final analysis was adjusted for clustering by centre to take this into account and results in a more robust estimate of standard errors, with a corresponding widening of the confidence intervals.

The main primary outcomes were compared by crude (unadjusted) odds ratios, with no imputation and also by odds ratios created by multiple logistic regression analysis adjusting for confounding factors using multiple imputation for missing values in the confounding variables. Note that if the $95 \% \mathrm{CI}$ of the odds ratio includes the value 1 , then there is no statistically significant difference between the treatment cohorts.

Secondary outcomes were tested using the chi-square statistic. Further treatment is required after UAE was investigated using life table methods and Kaplan-Meier survival curves.

\section{Results}

Of the 1734 eligible women, data could be collected for a total of 1122 women. However, no data were available for 14 women, four of whom were deceased, and these women were excluded from the final analysis. Data were therefore available for analysis on 1108 women (63.9\%) consenting or deceased women (hysterectomy, $n=459$ [60.2\%]; UAE, $n=649$ [66.8\%]). The average age at procedure was 46.5 years (SD 6.8) for the hysterectomy cohort and 43.8 years (SD 6.5) for the UAE cohort. The average length of follow up was 8.6 years (SD 3.4) for the hysterectomy cohort and 4.6 years (SD 2.0) for the UAE cohort. A minimum of 2 years of follow up was attained for $91.5 \%$ of the UAE cohort and $87.1 \%$ of the hysterectomy cohort. More than 5 years of follow up was attained for $46.5 \%$ of the UAE cohort and $86.7 \%$ of the hysterectomy cohort. Note that for some of the women who did not return their questionnaires, there may have been only short-term follow up available from their hospital notes. 
Table 1. Baseline characteristics

\begin{tabular}{|c|c|c|c|c|}
\hline & Hysterectomy $(n=459)$ & UAE $(n=649)$ & Total $(n=1108)$ & Significance* $(P$ value $)$ \\
\hline \multicolumn{5}{|l|}{ Demographic variables } \\
\hline Age (years) at procedure, mean ( $n$ ) SD & $46.5(459) 6.8$ & $43.8(649) 6.5$ & 44.9 (1108) 6.8 & $<0.0001$ \\
\hline \multicolumn{5}{|l|}{ Highest level of education } \\
\hline Primary & $150(32.7 \%)$ & $64(9.9 \%)$ & $214(19.3 \%)$ & \multirow[t]{4}{*}{$<0.0001$} \\
\hline Secondary & $138(30.1 \%)$ & $195(30.0 \%)$ & $333(30.1 \%)$ & \\
\hline Tertiary & $98(21.3 \%)$ & $320(49.3 \%)$ & $419(37.8 \%)$ & \\
\hline Missing & $73(15.9 \%)$ & $70(10.8 \%)$ & $142(12.8 \%)$ & \\
\hline \multicolumn{5}{|l|}{ Ethnicity } \\
\hline White & $382(83.2 \%)$ & $480(74.0 \%)$ & $862(77.8 \%)$ & \multirow[t]{4}{*}{$<0.0001$} \\
\hline Black & $6(1.3 \%)$ & $97(14.9 \%)$ & $103(9.3 \%)$ & \\
\hline Other & $9(2.0 \%)$ & $10(1.5 \%)$ & $19(1.7 \%)$ & \\
\hline Missing & $62(13.5 \%)$ & $62(9.6 \%)$ & $124(11.2 \%)$ & \\
\hline \multicolumn{5}{|l|}{ Parity } \\
\hline Nulliparous & $65(14.2 \%)$ & $296(45.6 \%)$ & $361(32.6 \%)$ & \multirow[t]{3}{*}{$<0.0001$} \\
\hline Multiparous & $391(85.2 \%)$ & $328(50.5 \%)$ & $719(64.9 \%)$ & \\
\hline Missing & $3(0.6 \%)$ & $25(3.9 \%)$ & $28(2.5 \%)$ & \\
\hline \multicolumn{5}{|l|}{ Menopausal status } \\
\hline Postmenopausal & $39(8.5 \%)$ & $35(5.4 \%)$ & $74(6.7 \%)$ & \multirow[t]{2}{*}{0.41} \\
\hline Not postmenopausal & $420(91.5 \%)$ & $614(94.6 \%)$ & $1034(93.3 \%)$ & \\
\hline Age (years) at menarche, mean (n) SD & $12.4(392) 1.6$ & $12.5(573) 1.5$ & $12.5(965) 1.5$ & 0.27 \\
\hline \multicolumn{5}{|l|}{ Medical-related variables } \\
\hline \multicolumn{5}{|l|}{ Smoking } \\
\hline Never & $235(51.2 \%)$ & $376(57.9 \%)$ & $611(55.1 \%)$ & \multirow[t]{3}{*}{0.006} \\
\hline Current/ex-smoker & $210(45.7 \%)$ & $238(36.7 \%)$ & $448(40.4 \%)$ & \\
\hline Missing & $14(3.1 \%)$ & $35(5.4 \%)$ & $49(4.4 \%)$ & \\
\hline \multicolumn{5}{|l|}{ Obesity (Obese—body mass index $\geq 30$ ) } \\
\hline Not obese & $257(56.0 \%)$ & $248(38.2 \%)$ & $505(45.6 \%)$ & \multirow[t]{3}{*}{0.92} \\
\hline Obese & $64(13.9 \%)$ & $63(9.7 \%)$ & $127(11.5 \%)$ & \\
\hline Missing & $138(30.1 \%)$ & $338(52.1 \%)$ & $476(43.0 \%)$ & \\
\hline \multicolumn{5}{|c|}{ Blood pressure (BP) (High BP_-diastolic $\geq 90 \mathrm{mmHg}$ and/or systolic $\geq 140 \mathrm{mmHg}$ ) } \\
\hline Normal & $242(52.7 \%)$ & $272(41.9 \%)$ & $514(46.4 \%)$ & \multirow[t]{3}{*}{$<0.0001$} \\
\hline High BP & $213(46.4 \%)$ & $114(17.6 \%)$ & $327(29.5 \%)$ & \\
\hline Missing & $4(0.9 \%)$ & $263(40.5 \%)$ & $267(24.1 \%)$ & \\
\hline \multicolumn{5}{|l|}{ Gynaecological co-morbidity } \\
\hline No & $404(88.0 \%)$ & $574(88.4 \%)$ & $978(88.3 \%)$ & \multirow[t]{2}{*}{0.83} \\
\hline Yes & $55(12.0 \%)$ & $75(11.6 \%)$ & $130(11.7 \%)$ & \\
\hline \multicolumn{5}{|l|}{ Medical co-morbidity } \\
\hline No & $322(70.2 \%)$ & $523(80.6 \%)$ & $845(76.3 \%)$ & \multirow[t]{2}{*}{$<0.0001$} \\
\hline Yes & $137(29.8 \%)$ & $126(19.4 \%)$ & $263(23.7 \%)$ & \\
\hline \multicolumn{5}{|l|}{ Prior pelvic surgery } \\
\hline No & $394(85.8 \%)$ & $480(74.0 \%)$ & $874(78.9 \%)$ & \multirow[t]{2}{*}{$<0.0001$} \\
\hline Yes & $65(14.2 \%)$ & $169(26.0 \%)$ & $234(21.1 \%)$ & \\
\hline \multicolumn{5}{|l|}{ Prophylactic antibiotics } \\
\hline No & $53(11.5 \%)$ & $169(26.0 \%)$ & $222(20.0 \%)$ & $<0.0001$ \\
\hline Yes & $406(88.5 \%)$ & $422(65.0 \%)$ & $828(74.7 \%)$ & \\
\hline Missing & $0(0.0 \%)$ & $58(8.9 \%)$ & $58(5.2 \%)$ & \\
\hline
\end{tabular}

Data were given as number (percentage) unless stated otherwise.

* Significance level $t$ test for continuous variables and chi-square for nonmissing categorical variables.

As anticipated, the two cohorts presented a different baseline profile for many of the a priori confounders (Table 1). These included statistically significant differences for age, educational level, ethnicity, parity, smoking, medical co- morbidity, prior pelvic surgery, high blood pressure and prophylactic antibiotics.

There were 341 complications identified in 234 women (hysterectomy, $n=120$; UAE, $n=114$ ). Information about 
complications was principally drawn from the clinical forms (based on hospital records), although there were 77 complications identified for 55 women from the patient questionnaires. Twenty-four of these were duplicates from complications already identified from the clinical forms, but a further 31 women reported one or more complications in their patient questionnaire that had not previously been identified (2.8\% of the women). The majority of these additional complications were identified among the UAE cohort, including requiring assistance with extraction of fibroids, infections and severe pain causing readmission to hospital. Of the 234 women who experienced complications, 165 women experienced only one, 49 experienced two and 20 experienced more than two complications. The numbers of complications by complication type are presented in Table 2. Where no complication was reported, either in the clinical or in the questionnaire, the woman was assumed to have experienced no complications.

Women categorised according to the highest level of complication experienced are presented in Table 3. The crude incidence of a priori defined complications (severe, major or minor) was higher for the hysterectomy cohort (26.1 versus $\left.17.6 \%, \chi_{(1)}^{2}, P=0.001\right)$. The crude incidence of severe/major complications was also higher for the hysterectomy cohort (11.3 versus $3.9 \%$ UAE, $\chi_{(1)}^{2}, P<0.0001$ ).

The unadjusted (crude) odds ratio for all complications for UAE versus hysterectomy was 0.60 (95\% CI 0.32-1.15). After adjusting for significant confounders, missing values and clustering by centre using multiple logistic regression (minimum model), the odds ratio was reduced to 0.48 (95\% CI $0.26-0.89$ ). The crude and adjusted treatment odds ratios and the confounding variables in the minimum model together with their 95\% CIs are presented on a log scale (treatment above the dashed line and confounders below) in Figure 1. All 95\% CIs are calculated after adjusting for clustering by centre.

In addition, it indicates the variables that may be important in determining whether a woman develops a complication (whose 95\% CIs do not contain one). Being obese, having a later onset of menarche, an existing medical co-morbidity, or having already undergone prior pelvic surgery raise the odds of experiencing a complication, while using prophylactic antibiotics at the time of the procedure reduces the odds of experiencing a complication. High blood pressure also appears to be slightly protective but may be an artefact of

Table 2. Numbers of complications by complication type

\begin{tabular}{|c|c|c|c|c|}
\hline $\begin{array}{l}\text { Severity of } \\
\text { complication }\end{array}$ & Type of complication & $\begin{array}{l}\text { Hysterectomy } \\
(n=459), n(\%)\end{array}$ & $\begin{array}{c}\text { UAE } \\
(n=649), n(\%)\end{array}$ & $\begin{array}{c}\text { Total } \\
(n=1108), n(\%)\end{array}$ \\
\hline \multirow[t]{7}{*}{ Severe } & Death & 0 & 0 & 0 \\
\hline & Pulmonary embolus & $3(0.7)$ & 0 & $3(0.3)$ \\
\hline & Myocardial infarction & 0 & 0 & 0 \\
\hline & Cerebrovascular accident (stroke) & 0 & 0 & 0 \\
\hline & Organ failure & $1(0.2)$ & 0 & $1(0.1)$ \\
\hline & Other severe & $1(0.2)$ & $1(0.2)$ & $2(0.2)$ \\
\hline & Total severe & $5(1.1)$ & $1(0.2)$ & $6(0.5)$ \\
\hline \multirow[t]{8}{*}{ Major } & Permanent amenorrhoea ( $<40$ years) & 0 & $1(0.2)$ & $1(0.1)$ \\
\hline & Radiation burn & 0 & 0 & 0 \\
\hline & Blood transfusion required & $34(7.4)$ & $4(0.6)$ & $38(3.4)$ \\
\hline & Structural damage caused by treatment & $16(3.5)$ & $5(0.8)$ & $21(1.9)$ \\
\hline & $\begin{array}{l}\text { Septicaemia, emergency myomectomy/ } \\
\text { hysterectomy }\end{array}$ & $2(0.4)$ & $17(2.6)$ & $19(1.7)$ \\
\hline & Thrombosis & $2(0.4)$ & 0 & $2(0.2)$ \\
\hline & Other major & $9(2.0)$ & $1(0.2)$ & $10(0.9)$ \\
\hline & Total major & $63(13.7)$ & $28(4.3)$ & $91(8.2)$ \\
\hline \multirow[t]{8}{*}{ Minor } & Minor infections ( $<30$ days) & $62(13.5)$ & $38(5.9)$ & $100(9.0)$ \\
\hline & Haematoma requiring treatment & $6(1.3)$ & $4(0.6)$ & $10(0.9)$ \\
\hline & Adverse drug reaction & $3(0.7)$ & $8(1.2)$ & $11(1.0)$ \\
\hline & Permanent amenorrhoea ( $\geq 40$ years) & $1(0.2)^{\star}$ & $9(1.4)$ & $10(0.9)$ \\
\hline & Retention of urine requiring catheterisation & $10(2.2)$ & $9(1.4)$ & $19(1.7)$ \\
\hline & Fibroid extraction requiring assistance & 0 & $41(6.3)$ & $41(3.7)$ \\
\hline & Other minor ( $<30$ days) & $26(5.7)$ & $27(4.2)$ & $53(4.8)$ \\
\hline & Total minor & $108(23.5)$ & $136(21.0)$ & $244(22.0)$ \\
\hline Total complications & & $176(38.3)$ & $165(25.4)$ & $341(30.8)$ \\
\hline
\end{tabular}

*This woman had a subtotal hysterectomy. 
Table 3. Complication severity by treatment

\begin{tabular}{lccc}
\hline $\begin{array}{l}\text { Complication } \\
\text { type }\end{array}$ & $\begin{array}{c}\text { Hysterectomy, } \\
\boldsymbol{n ( \% )}\end{array}$ & $\begin{array}{c}\text { UAE, } \\
\boldsymbol{n}(\%)\end{array}$ & $\begin{array}{c}\text { Total, } \\
\boldsymbol{n}(\%)\end{array}$ \\
\hline All complications & $120(26.1)$ & $114(17.6)$ & $234(21.1)$ \\
Severe & $3(0.7)$ & $1(0.2)$ & $4(0.4)$ \\
Major & $49(10.7)$ & $24(3.7)$ & $73(6.6)$ \\
Minor & $68(14.8)$ & $89(13.7)$ & $157(14.2)$ \\
None & $339(73.9)$ & $535(82.4)$ & $874(78.9)$ \\
Total & $459(100)$ & $649(100)$ & $1108(100)$ \\
\hline
\end{tabular}

the recording and categorisation of this variable. The increased risk for the 'other' ethnic group may be due to the small number of such women present in the HOPEFUL study and would need to be investigated by further studies.

The unadjusted (crude) odds ratio of severe or major complications, comparing UAE with hysterectomy, was 0.31 ( $95 \%$ CI $0.15-0.66$ ). This was reduced to 0.25 (95\% CI $0.13-0.48$ ) after adjustment for confounding and clustering by centre. This odds ratio is smaller than for all complications, indicating that the extra risk associated with undergoing a hysterectomy is primarily associated with severe and/or major complications. Investigation into the actual complications shows that the excess in the hysterectomy group was mainly attributable to an increased incidence of the need for blood transfusion (7.4 versus $0.4 \%$ ) and structural damage (3.5 versus $0.8 \%$ ). Women undergoing UAE may still experience complications, but they are more likely to be of minor severity.

Being obese, or having an existing medical co-morbidity raises the odds of experiencing a severe/major complication, while using prophylactic antibiotics at the time of the pro- cedure reduces the odds, although this is not as strong as for all complications. The increased risk for the 'other' ethnic group has all but disappeared, possibly due to the smaller numbers of women in this ethnic group who had severe/ major complications.

Anticipated GSEs of UAE included chronic, although selflimiting vaginal discharge $(12.6 \%)$, spontaneous fibroid expulsions (7.6\%) and postembolisation syndrome (17.7\%). These were reported by $32.7 \%$ of the UAE women. Some women $(8.9 \%)$ experienced both complications and GSEs. Half of these had GSEs that were independent of their complications and the remaining half had GSEs that were associated with their complications. The majority of these latter were chronic discharge caused by infection of disintegrating fibroids, chronic discharge leading to assisted fibroid extraction or both. In total, $41.3 \%$ of the UAE cohort experienced some adverse effects (complications and/or side effects) of their treatment, although most were side effects that the women had been informed about prior to their UAE. The duration of these side effects varied from a matter of a few hours to persisting for several months. Multiple logistic modelling, adjusting for complications and confounders, found that prophylactic antibiotics were also protective against GSEs in addition to being protective for both cohorts against complications. Reporting of side effects varied widely between centres.

Secondary outcomes concerning efficacy were available retrospectively from patient questionnaires $(n=986$; hysterectomy, $n=397$; UAE, $n=589$ ) (Table 4 ). The general health of both cohorts was similar at baseline. Of those women completing questionnaires, $75 \%$ of the hysterectomy cohort reported improved health status (average 9 years postsurgery) compared with $65 \%$ of the UAE cohort (average 5 years) $(P<$ $0.0001)$. For those completing the questions on 'feelings about

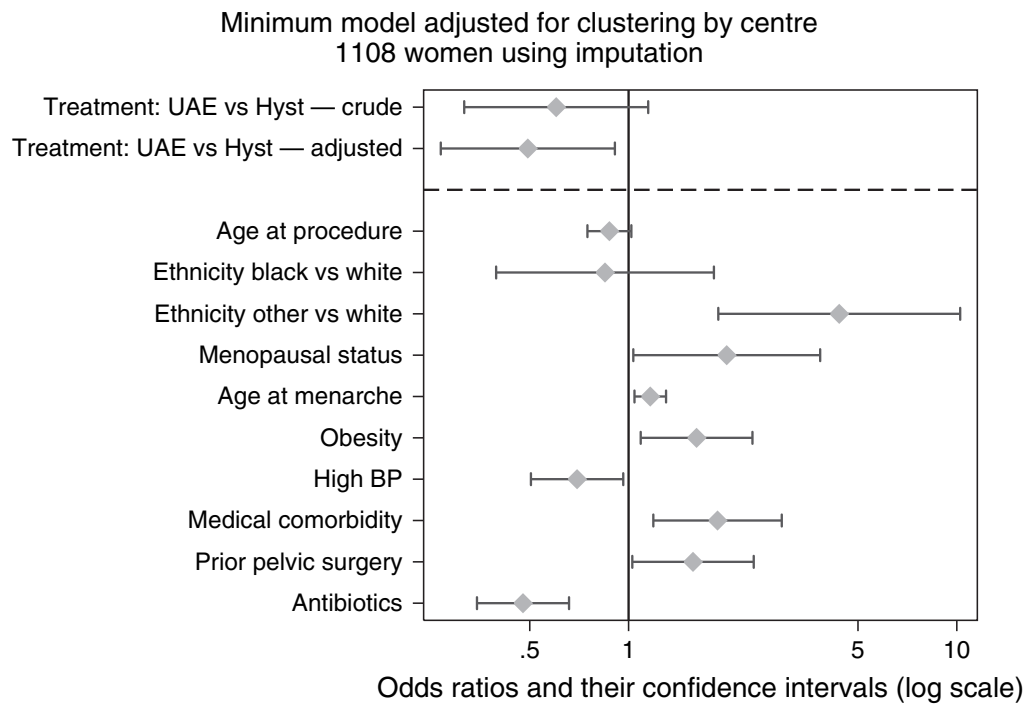

Figure 1. Primary outcome, complications versus no complications: odds ratios for minimum model. 
your treatment', the hysterectomy cohort were significantly different than the UAE cohort in 'relief of fibroid symptoms' (99.2 versus $85.2 \%, P<0.0001$ ), and 'feeling better since their index treatment' (95.8 versus $83.8 \%, P<0.0001$ ) was also significantly different for the hysterectomy cohort compared with the UAE cohort. However, paradoxically, only $85.5 \%$ of the hysterectomy cohort would recommend their treatment to a friend compared with $91.4 \%$ of the UAE cohort $(P=0.007)$.

The expectations of the UAE cohort were less likely to be fulfilled $(73.5 \%$ UAE versus $93.5 \%$ hysterectomy, $P<0.0001$ ), possibly due in part to the high expectations of women in the (early) UAE cohort. There was no difference between the cohorts for those who reported no problems caused by the treatment (79.5\% UAE versus $75.4 \%$ hysterectomy, $P=0.18$ ). From the free text analysis of expectations not fulfilled, it is apparent that the management of expectations is particularly important for the UAE cohort. Many of the women were self-referred and had high, maybe unrealistic expectations, some choosing UAE in the hope of achieving pregnancy. ${ }^{20}$

One hundred and nineteen women $(18.3 \%)$ in the UAE cohort underwent a further 134 treatments for fibroids, 4.5\% further UAE, $4.9 \%$ myomectomy and $11.2 \%$ hysterectomy. This includes $3.9 \%$ requiring hysterectomies during the first year after UAE, $1.1 \%$ due to complications (as defined in Table 2), mainly due to severe pain or sepsis. Figure 2 shows the cumulative percentage of women to their first further fibroid treatment after their initial index treatment. After adjusting for differential time of follow up using survival analysis to first further treatment, the UAE women had a 23\% (95\% CI 19-27\%) chance of requiring further treatment for fibroids (or complications) within the first 7 years.

In the UAE cohort, 319 (49.2\%) women indicated on their questionnaire that at the time of their treatment, they did not want children in the future, $187(28.8 \%)$ indicated a hope to have any/more children and 22\% were uncertain or did not complete this question. Twenty-seven women achieved 37 pregnancies after UAE (Table 5). This represents $4.2 \%$ of the total UAE cohort, which is $8.5 \%$ of the $303(58.2 \%)$ women who indicated that they wished or were uncertain of their wish for children. The average age at pregnancy was 38 years (SD 3.3) at an average of 3 years after UAE. Of the women who achieved pregnancies, $78 \%$ were nulliparous and $74 \%$ expressed a wish to have any/more children. Of the 37 pregnancies, there were 15 miscarriages, 2 ectopic pregnancies, 1 termination and 19 successful live births (from 16 [2.3\%] women). Seventy-nine percent of the live births were delivered by caesarean section, six due to complications of pregnancy or delivery.

\section{Discussion}

Hysterectomy involves major surgery and has associated risks. Women stay in hospital for on average 5 days and then may take up to 3 months to recuperate. However, once the uterus is removed, there is no recurrence of fibroid-related symptoms. In this study, three $(0.8 \%)$ women reported symptoms after hysterectomy, which may be related to subtotal hysterectomy or residual pelvic pain.

Table 4. Secondary outcomes (efficacy) from patient questionnaire

\begin{tabular}{|c|c|c|c|c|}
\hline Satisfaction with index treatment & Hysterectomy, $n$ (\%) & UAE, $n(\%)$ & Total $(n=986) n(\%)$ & Significance* ( $P$ value) \\
\hline \multicolumn{5}{|l|}{ Symptoms relieved } \\
\hline Yes & $352(99.2)$ & $472(85.2)$ & $824(90.6)$ & $<0.0001$ \\
\hline No & $3(0.8)$ & $82(14.8)$ & $85(9.4)$ & \\
\hline \multicolumn{5}{|l|}{ Felt better since treatment } \\
\hline Yes & $320(95.8)$ & $434(83.8)$ & $754(88.5)$ & $<0.0001$ \\
\hline No & $14(4.2)$ & $84(16.2)$ & $98(11.5)$ & \\
\hline \multicolumn{5}{|c|}{ Would have repeat treatment for fibroids } \\
\hline Yes & $279(85.1)$ & $473(86.6)$ & $752(86.0)$ & 0.52 \\
\hline No & $49(14.9)$ & $73(13.4)$ & $122(14.0)$ & \\
\hline \multicolumn{5}{|l|}{ Recommend to a friend } \\
\hline Yes & $278(85.5)$ & $510(91.4)$ & $788(89.2)$ & 0.007 \\
\hline No & $47(14.5)$ & $48(8.6)$ & $95(10.8)$ & \\
\hline \multicolumn{5}{|l|}{ Expectations fulfilled } \\
\hline Yes & $343(93.5)$ & $417(73.5)$ & $760(81.4)$ & $<0.0001$ \\
\hline No & $24(6.5)$ & $150(26.5)$ & $174(18.6)$ & \\
\hline \multicolumn{5}{|c|}{ Reported problems caused by treatment } \\
\hline No & $221(75.4)$ & $388(79.5)$ & $609(78.0)$ & 0.18 \\
\hline Yes & $72(24.6)$ & $100(20.5)$ & $172(22.0)$ & \\
\hline
\end{tabular}

${ }^{*} \chi_{(1)}^{2}$ test on nonmissing data. 


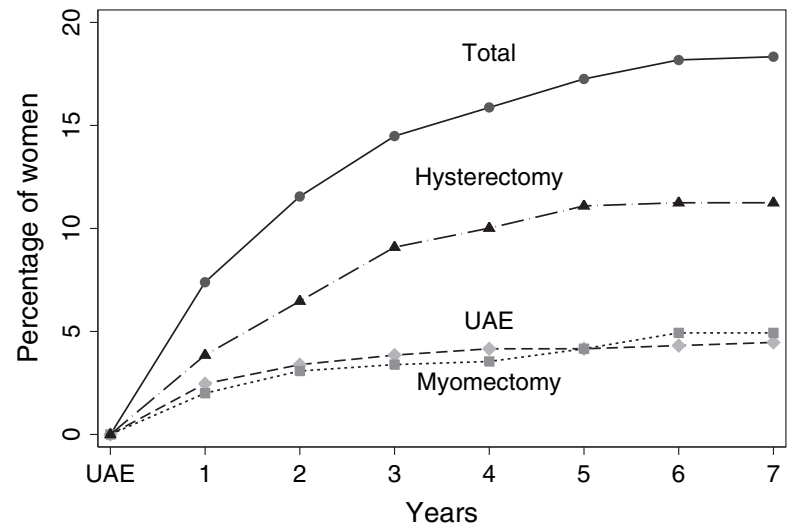

Figure 2. The cumulative percentage of women experiencing their first fibroid treatment subsequent to their index UAE.

UAE is a minimally invasive technique, and the great majority of women remain in hospital overnight to enable appropriate pain management. There were fewer complications experienced by the women in the UAE cohort, in particular the more severe/major complications, comparable with other studies of short-term outcomes of UAE and hysterectomy. ${ }^{6-16}$

GSEs are expected with embolisation. These include postembolisation syndrome, which may last up to 10 days and is the justification for advising women to take appropriate time off work. Fibroid expulsion is another GSE where infarcted fibroids, particularly submucosal fibroids, are expelled per vagina and most do not require treatment. If assistance, including further hospital treatment was required, this was classified as a complication.

Further research is required to confirm an apparent benefit of prophylactic antibiotics in reducing complications in both cohorts and GSEs in the UAE cohort. The effect of prophylactic antibiotics observed in this study may be caused by differential use of antibiotics by different centres and also by differential reporting of GSEs by different centres.

Lack of resolution or recurrence of fibroid-related symptoms following UAE may lead to further treatments for fibroids (repeat UAE, myomectomy or hysterectomy). The reasons for this are likely to be related to the size and position of the fibroid and the technical difficulties during embolisation. The fibroid registry in the USA has suggested that UAE for $10 \mathrm{~cm}$ submucosal fibroids causing menorrhagia results in better outcome than for larger intramural or subserosal fibroids causing bulk symptoms. ${ }^{15}$ Inadequate embolisation of fibroids can occur due to spasm of the uterine artery or aberrant arterial supply. Often such aberrant blood supplies are through the ovarian arteries, and while these can be embolised, and sometimes are in older women, in younger women, there is the increased risk of early menopause and women will often decline this further treatment.
Women in the HOPEFUL study had a 23\% chance (95\% CI $19-27 \%$ ) of requiring further treatment after adjusting for differential time of follow up. A hysterectomy was required by $3.9 \%$ within the first year (comparable with the findings of REST) ${ }^{10,11}$ and a further $7.4 \%$ from 2 to 7 years after UAE.

Consideration should be given to the fact that the UAE procedures in HOPEFUL were carried out at a time when experience was limited and pain control regimes not fully effective. ${ }^{28}$ This may no longer be the case, as experience and pain control are more sophisticated and in most centres pain management teams are involved in postprocedure pain relief. However, for the reasons cited, symptomatic relief may not always be possible and a hysterectomy may become necessary.

The uterus is preserved after UAE and hence also the potential for future fertility. Pregnancy is possible after UAE, although it is not recommended due to possible unknown risks during pregnancy and parturition. ${ }^{19}$ The HOPEFUL study adds some information on pregnancies after UAE, but we have no precise information on how many women were trying to conceive after embolisation, so we cannot calculate or interpret a 'success rate' from our data. The HOPEFUL pregnancy rate (excluding women who expressly did not want pregnancy) of $8.5 \%$ compares well with other studies, where the pregnancy rates in those desiring pregnancy are reported as $3.8,{ }^{32} 4.3^{33}$ and $5.3 \% .{ }^{34}$ In all these studies, miscarriage and caesarean rates were similar to those presented in HOPEFUL. It is interesting to note that four women in HOPEFUL had both live births and miscarriages. Further research is required to identify whether myomectomy or UAE should be the treatment of choice for women with fibroids desiring pregnancy.

\section{Conclusions}

There is a paucity of data on longer term follow up of women with symptomatic fibroids treated by UAE. There is also a lack of large studies comparing UAE with more established treatments. This study contributes data on safety and efficacy of UAE and hysterectomy over the medium term by using a pragmatic study design incorporating multivariate statistical methodology to minimise biases where possible.

The women who underwent UAE reported here, represent experience of the very early days of the new procedure so may provide conservative results. The evidence accumulated in this study strongly suggests UAE is a safe procedure over the medium term with fewer significant complications than hysterectomy and has an important role in the effective treatment of symptomatic fibroids. Although one-quarter of women undergoing UAE may require subsequent treatment for continuing symptoms, UAE still remains cost-effective. ${ }^{21}$ While this procedure enables preservation of fertility, caution 
Table 5. A summary of pregnancies after UAE including baseline characteristics and stated aspirations

\begin{tabular}{|c|c|c|c|c|c|c|c|c|}
\hline ID* & Date UAE & Age & Parity & Fertility** & Event $1 * * *$ & Event 2*** & Event $3 * * *$ & Event $4 * * *$ \\
\hline 1 & 1999 & 36 & 0 & Unk & Ectopic (early) 2001 & & & \\
\hline 2 & 1999 & 39 & 0 & Yes & Misc & & & \\
\hline 3 & 2000 & 33 & 1 & Yes & Misc (20) 2000 & Live (N) 2002 & & \\
\hline 4 & 2000 & 41 & 1 & Yes & Misc & & & \\
\hline 5 & 1998 & 36 & 0 & Yes & Live $(C / S)$ & & & \\
\hline 6 & 1999 & 35 & 0 & Yes & Misc & & & \\
\hline 7 & 1997 & 38 & 0 & Yes & Live (C/S) 1999 & & & \\
\hline 8 & 1999 & 35 & 0 & Unk & Live (C/S)(35) 2001 & & & \\
\hline 9 & 2000 & 35 & 1 & Unk & Live (C/S) (37) 2004 & & & \\
\hline 10 & 2001 & 34 & 0 & Yes & Misc & & & \\
\hline 11 & 1999 & 37 & 1 & Yes & Misc & & & \\
\hline 12 & 1998 & 40 & 1 & Yes & Misc (9) & Live (C/S) 2000 & & \\
\hline 13 & 1998 & 32 & 0 & Yes & Live (C/S) (39) 2000 & & & \\
\hline 14 & 1999 & 34 & 0 & Yes & Live (C/S) 2005 & & & \\
\hline 15 & 1998 & 35 & 0 & No & Live (C/S) 2000 & Live (C/S) 2004 & & \\
\hline 16 & 2000 & 43 & 0 & Yes & $\operatorname{Misc}(10)$ & & & \\
\hline 17 & 1999 & 34 & 1 & Yes & Live (C/S) (39) 2000 & & & \\
\hline 18 & 1999 & 46 & 0 & Yes & TOP 2001 & & & \\
\hline 19 & 2002 & 43 & 0 & No & Live (C/S) (38) 2003 & & & \\
\hline 20 & 1999 & 29 & 0 & Yes & Live (C/S) (40) 2000 & & & \\
\hline 21 & 2000 & 38 & 0 & Yes & $\operatorname{Misc}(6)$ & & & \\
\hline 22 & 1997 & 34 & 0 & Unk & Live (N) (41) 2001 & & & \\
\hline 23 & 1998 & 28 & 0 & Other & Live (C/S) (42) 2001 & Live (N) 2004 & & \\
\hline 24 & 1998 & 30 & 0 & Yes & Live (C/S) (38) 2001 & Misc (8) 2005 & & \\
\hline 25 & 1997 & 40 & 0 & Other & Misc (5) & & & \\
\hline 26 & 1998 & 32 & 0 & Yes & Ectopic (6) 2001 & Misc (5) 2002 & Misc (6) 2003 & \\
\hline 27 & 1998 & 35 & 0 & Yes & Live (C/S) (38) 2000 & Misc (12) 2001 & Misc (11) 2001 & Live (N) (40) 2002 \\
\hline
\end{tabular}

Misc, miscarriages; TOP, termination of pregnancy.

Summary: age at first pregnancy after UAE $=37.6$ years $(S D=3.3), 77.8 \%$ nulliparous, $74.1 \%$ hoped to have any/more children .

*Patient IDs have been replaced to preserve anonymity.

**Fertility aspirations: yes, hoped to have any/more children; no, did not want any/more children; other, not sure; Unk, no questionnaire of not filled in.

$\star * \star$ Event: birth type (if live: type of delivery-C/S, caesarean; N, natural) (number of weeks of gestation, if known) year of birth.

still needs to be exercised with regard to counselling women still desirous of pregnancies.

Although not a randomised study, the aggregate conclusions from this study of contemporary practice are as robust as they can be at this stage in the development of a new technique for treating symptomatic fibroids. This study provides information about potential risks and benefits that will enable clinicians to counsel women more accurately. The qualitative analysis of women's experience is particularly useful in this respect. ${ }^{20}$

\section{Contribution to authorship}

K.McP. was chief investigator for the HOPEFUL study and is guarantor. S.D. contributed to the design of the study, created and managed the study database, was responsible for statistical analysis and interpretation and contributed to writing the report in conjunction with A.H. A.H. contributed to the design of the HOPEFUL study, managed the conduct of the study, carried out the evidence literature review, contributed to the interpretation of the data and was primarily responsible for writing all sections of the report. T.N. and M.M. contributed to the design of the study and provided advice on all the clinical aspects of the study. All authors approved the final version.

\section{Acknowledgements}

We thank Carol Edwards (Qualitative Focus and University of Leicester) and Lara Waldenmaier (Oxford) for their work on the free text analyses, and Andrew Briggs and Olivia $\mathrm{Wu}$ (Health Economics, Glasgow) who carried out all the economic analyses. Thank you to all the hospital centres 
participating in HOPEFUL who provided clinical data for their consenting women (Appendix S1). This study would not have been possible without the valued contributions of the women who gave consent for us to collect information from their hospital records and who gave their time to share their experiences of their fibroids and treatments. The full monograph of the project HTA Ref: 03/60/01 'The HOPEFUL study: a multicentre retrospective cohort study comparing the efficacy, safety and cost-effectiveness of hysterectomy and UAE for the treatment of symptomatic uterine fibroids' is estimated to be published by February 2008. The HTA monograph is protected by Crown copyright. This paper differs from the full monograph by presenting a summary of the important quantitative findings on the medium-term safety and efficacy of the two treatments.

\section{Supplementary material}

The following supplementary material is available for this article:

Appendix S1. Details of collaborators and members of the Trial Steering Committee.

This material is available as part of the online article from: http://www.blackwell-synergy.com/doi/abs/doi: 10.1111/j.14710528.2007.01526.x.

(This link will take you to the article abstract).

Please note: Blackwell Publishing are not responsible for the content or functionality of any supplementary materials supplied by the authors. Any queries (other than missing material) should be directed to the corresponding author for the article.

\section{References}

1 Marshall LM, Spiegelman D, Barbieri RL, Goldman MB, Manson JE, Colditz GA, et al. Variation in the incidence of uterine leiomyoma among premenopausal women by age and race. Obstet Gynecol 1997;90:967-73.

2 Flake GP, Andersen J, Dixon D. Etiology and Pathogenesis of uterine leiomyomas: a review Environ Health Perspect 2003;111:1037-54.

3 Lethaby A, Vollenhoven B. Fibroids (uterine myomatosis, leiomyomas). Am Fam Physician 2005;71:1753-6.

4 Ravina JH, Herbreteau D, Ciraru-Vigneron N, Bouret JM, Houdart H, Aymard A, et al. Arterial embolisation to treat uterine myomata. Lancet 1995;346:671-2.

5 Gupta JK, Sinha AS, Lumsden MA, Hickey M. Uterine artery embolization for symptomatic uterine fibroids. Cochrane Database Syst Rev 2006: CD005073. DOI: 10.1002/14651888.CD005073.pub2.

6 Pinto I, Chimeno P, Romo A, Paul L, Haya J, de la Cal MA, et al. Uterine fibroids: uterine artery embolisation versus abdominal hysterectomy for treatment a prospective, randomised and controlled clinical trial. Radiology 2003;226:425-31.

7 Hehenkamp WJK, Volkers NA, Donderwinkel PFJ, de Blok S, Birnie E, Ankum WA, et al. Uterine artery embolization versus hysterectomy in the treatment of symptomatic uterine fibroids (EMMY trial): peri-and post-procedural results from a randomized controlled trial. Am J Obstet Gynecol 2005;193:1618-29.

8 Volkers NA, Hehenkamp WJ, Birnie E, de Vries C, Holt C, Ankum WM, et al. Uterine artery embolization in the treatment of symptomatic uterine fibroid tumors (EMMY trial): peri procedural results and complications. J Vasc Interv Radiol 2006;17:471-80.

9 Hehenkamp WJ, Volkers NA, Birnie E, Reekers JA, Ankum WM. Clinical investigations: pain and return to daily activities after uterine artery embolization and hysterectomy in the treatment of symptomatic uterine fibroids: results from the randomized EMMY trial. CardiovasC Intervent Radiol 2006;29:179-87.

10 Moss JG. Randomised comparison of uterine artery embolisation with surgical treatment in patients with fibroids (REST trial): one year results. Presented at CIRSE (Cardiovascular and Interventional Radiology Society of Europe), September 2006, Rome, Italy. Conference Abstracts, pp 94-95.

11 The REST Investigators. Uterine-artery embolization versus surgery for symptomatic uterine fibroids. N Engl J Med 2007;353:360-70.

12 The FIBROID Registry: Report of Structure, Methods, and Initial Results (Internet Citation). AHRQ Publication No. 05(06)-RG008, October 2005. Rockville, MD: Agency for Healthcare Research and Quality, [www.ahrq.gov/research/fibroid/] Accessed 12 September 2006.

13 Myers ER, Goodwin S, Landow W, Mauro M, Peterson E, Pron G, et al. Prospective data collection of a new procedure by a specialty society: the FIBROID registry. Obstet Gynecol 2005;106:44-51. Erratum: Obstet Gynecol 2005;106:869.

14 Worthington-Kirsch R, Spies JB, Myers ER, Mulgund J, Mauro M, Pron $\mathrm{G}$, et al. FIBROID Investigators. The Fibroid Registry for outcomes data (FIBROID) for uterine embolization: short-term outcomes. Obstet Gynecol 2005;106:52-9. Erratum: Obstet Gynecol 2005;106 (4):869.

15 Spies JB, Myers ER, Worthington-Kirsch R, Mulgund J, Goodwin S, Mauro $\mathrm{M}$, et al. The FIBROID registry: symptom and quality-of-life status 1 year after therapy. Obstet Gynecol 2005;106:1309-18.

16 Coleman P, Ayiku L, Nicholl J, Cross E. Systematic review of the efficacy and safety of uterine artery embolisation in the treatment of fibroids. July 2004. School of Health and Related Research, Sheffield. 'Review Body' for the National Institute for Health and Clinical Excellence (NICE) Interventional Procedures Programme (www.nice. org.uk). 2004 [www.nice.org.uk/ip020systematicreview]. Accessed 12 September 2006.

17 UK Clinical Guidance, National Institute for Health and Clinical Excellence (NICE). IPG094 Uterine artery embolisation for the treatment of fibroids-guidance. 2004 [www.nice.org.uk/IPG094]. Accessed 12 September 2006.

18 UK Clinical Guidance, National Institute for Health and Clinical Excellence (NICE). IPG094 Uterine artery embolisation for the treatment of fibroids-information for women considering the procedure, and for the public [www.nice.org.uk/IPG094publicinfo]. Accessed 12 September 2006.

19 Royal College of Radiologists and Royal College of Obstetricians and Gynaecologists. Report of a Joint Working Party. Clinical Recommendations on the Use of Uterine Artery Embolisation in the Management of Fibroids. (Royal College Joint Report) RCOG Press, 2000. [www.rcog.org.uk/resources/public/pdf/embolisation.pdf].

20 Hirst A, Dutton S, Wu O, Briggs A, Edwards C, Waldenmaier L, et al. The HOPEFUL Study: a multi-centre retrospective cohort study comparing the efficacy, safety and cost-effectiveness of hysterectomy and uterine artery embolisation (UAE) for the treatment of symptomatic uterine fibroids. NHS Health Technology Assessment Programme. HTA Ref: 03/60/01 Estimated publication date March 2008 (in press). [www.hta.nhsweb.nhs.uk].

21 Wu O, Briggs A, Dutton S, Hirst A, Maresh M, Nicholson T, et al. Uterine artery embolisation or hysterectomy for the treatment of symptomatic 
uterine fibroids: a cost-effectiveness analysis of the HOPEFUL study. BJOG 2007;114:1352-62.

22 Maresh M, Metcalfe MA, McPherson K, Overton C, Hall V, Hargreaves $J$, et al. The VALUE national hysterectomy study: description of the patients and their surgery. BJOG 2002;109:302-12.

23 Ryan JM. Misinterpretation of postembolisation syndrome after conservative treatment of fibroids. J Vasc Interv Radiol 2004;15:99-100.

24 Payne JF, Haney AF. Serious complications of uterine artery embolization for conservative treatment of fibroids. Fertil Steril 2003;79: 128-31.

25 Bissler JJ, Racadio J, Donnelly LF, Johnson ND. Reduction of postembolization syndrome after ablation of renal angiomyolipoma. Am J Kidney Dis 2002;39:966-71.

26 Leung DA, Goin JE, Sickles C, Raskay BJ, Soulen MC. Determinants of postembolization syndrome after hepatic chemoembolization. J Vasc Interv Radiol 2001;12:321-6.

27 Patel NH, Hahn D, Rapp S, Bergan K, Coldwell DM. Hepatic artery ermbolization: factors predisposing to postembolization pain and nausea. J Vasc Interv Radiol 2000;11:453-60.
28 Siskin GP, Bonn J, Worthington-Kirsch RL, Smith SJ, Shlansky-Goldberg $R$, Machan LS, et al. Uterine fibroid embolization: pain management. Tech Vasc Interv Radiol 2002;5:35-43.

29 StatCorp. Stata Statistical Software: Release 8.2 (Intercooled). College Station, TX: Stata Press, 2003.

30 McPherson K, Metcalfe MA, Herbert A, Maresh M, Casbard A, Hargreaves J, et al. Severe complications of hysterectomy: the VALUE study. BJOG 2004;111:688-94.

31 Rubin DB. Inference and missing data. Biometrika 1976;63:581-92.

32 Pron G, Mocarski E, Bennett J, Vilos G, Common A, Vanderburgh L, et al. Pregnancy following uterine artery embolisation for leiomyomata: the Ontario multicentre study. Obstet Gynecol 2005;105: 67-76.

33 Walker WJ, McDowell SJ. Pregnancy after uterine artery embolisation for leiomyomata: a series of 56 completed pregnancies. Am J Obstet Gynecol 2006;195:1266-71.

34 Kim MD, Kim NK, Kim HJ, Lee MH. Pregnancy following uterine artery embolisation with polyvinyl alcohol particles for patients with uterine fibroid or adenomyosis. Cardiovasc Intervent Radio/2005;28:611-15. 